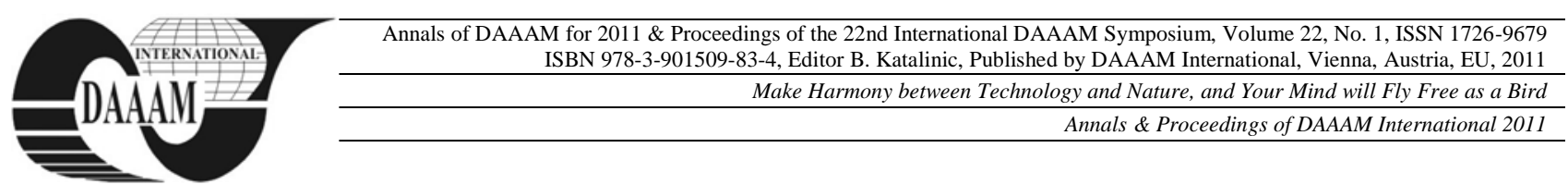

\title{
DISTILLATION COLUMN MODEL INTRODUCTION
}

\author{
KACZMARCZYK, V[aclav]; SIR, M[ichal]; BRADAC, Z[denek] \& STOHL, R[adek]
}

\begin{abstract}
This article introduces the model of distillation column for the steam distillation. After the introduction of a steam distillation process, the model description and list of actuators and sensors is mentioned and described. In the last chapter brief overview of model control is shown

Key words: distillation column, distillation, ModbusTCP, ASInterface, chemical process
\end{abstract}

\section{INTRODUCTION}

This article is mentioned as an introduction article to the distillation column model control and visualization project. The model is designed for steam distillation, so the second chapter is focused mainly to this kind of distillation. The model is controlled by PLC. Necessary actuators and sensors are connected to it through AS-Interface input/output modules. The PLC realizes only basic safety loops. The supervisor control is implemented through the ModbusTCP communication protocol. The model is primarily constructed to be a learning aid for students of the department of Control and Instrumentation of the author's parent faculty. The idea of twolevel control is thus perfectly reasonable. The PLC constantly measures model state variables and performs simple safety loops. If there is any supervisor demand to change the model state, the desired value (target actuator state) is firstly compared within the PLC with its own algorithm and only in case PLC decides the demand is safe and coherent, it is enabled and performed. The PLC has thus always higher decision priority.

\section{DISTILLATION}

Distillation belongs to separation methods group (Churáček, 2007). These methods are used to separate samples to at least two parts. The aim of the distillation is to increase the concentration of one selected ingredient of the compound. The method is based on different ingredient fugacity (different boiling temperature). When heated up, the part of the mixture transforms to steam, in the separated part of column condensates again and the product including more volatile components is created. In the distillation residue (the part that has not been vaporized) remain less volatile components.

\subsection{Steam distillation (Allbright, 2009)}

When the water steam is used for the distillation, the distilled mixture boiling temperature is decreasing (to the temperature corresponding state, when the sum of the steam pressure and mixture vapor pressure equals atmospheric pressure). This distillation kind can be used only for materials that are not soluble with the water.

Steam distillation is often used on the filed of organic synthesis for cleaning of liquids with relatively high boiling point, or liquids that spontaneously reduce when reaching the boiling point. There are several reasons why to use the water steam:
- Organic compound are essentially immiscible with water in the liquid phase.

- The steam amount necessary to create specific amount of the substance is quite small because of relatively low molar weight compared to majority of organic compound.

- The tension of the water steam significantly decreases the boiling point of mixture.

For the steam distillation, the steam dispenser is necessary. Before the distillation process can start, the dispenser and the distillation jar should be heated to almost $100{ }^{\circ} \mathrm{C}$ (steam condensing back to water should not increase volume of distilled mixture). When the water in the dispenser starts boiling, the steam can be released and the distillation starts.

\section{DISTILLATION COLUMN MODEL}

The model is intended to learn practical skills in the field of technological process control. The volume of both containers the dispenser and the distillation jar is 4 liters, so model is relatively small. Despite of its size, the model safety must be taken into account. When during operation, the model can contain almost 8 liters of water (and mixture) with temperature of $100{ }^{\circ} \mathrm{C}$. There exists serious danger for electrical devices and operator in case of water leakage, steam emission, or an explosion. While electrical devices are either shielded or placed into switchboard box, the danger for people can not be underestimated. Safety subsystems can be divided to active and passive:

- Passive subsystems: passive overpressure system in the dispenser.

- Active subsystems: active overpressure system in the dispenser, temperature and water-level sensor in the dispenser, temperature, level and pressure sensor in the distillation jar.

All mentioned sensors are used for the distillation process control as well. In the following, the way how information from these sensors is processed, is described. The model is depicted in the figure 1 .

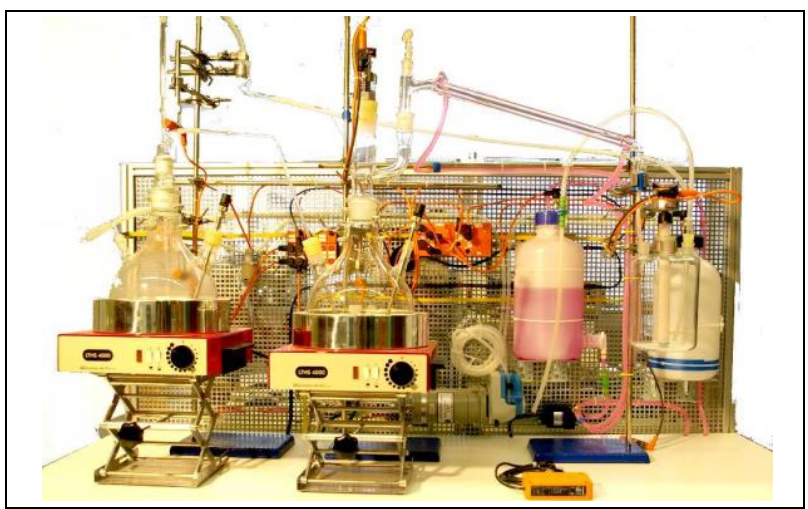

Fig. 1. Present state of the model 


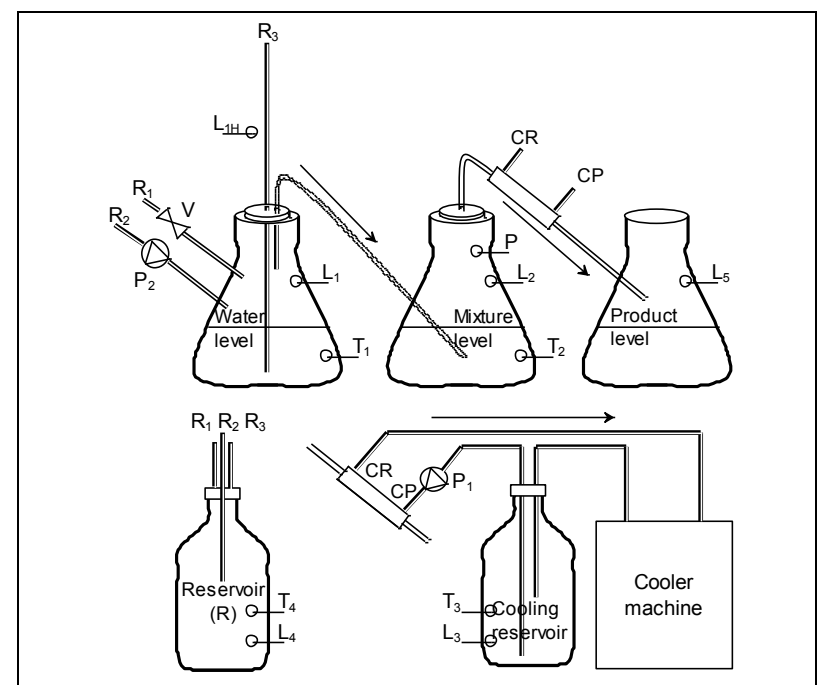

Fig. 2. Technological scheme of the model

Above mentioned safety subsystems always consist of control loops. These loops (both passive and active) are briefly shown here. If any of bellow mentioned situations occur, heaters $\mathrm{H} 1$ and $\mathrm{H} 2$ are turned down until the breakdown is removed (and reset by the supervisor control):

- When pressure in the dispenser increases, to water flows through tube R3 to the reservoir R (passive system). The level sensor $\mathrm{L} 1 \mathrm{H}$ is activated (active system). This passive system is crucial in case that any other system fails.

- When pressure in distillation jar increases (sensor P).

- When temperature in the dispenser increases (T1).

- When temperature in the distillation jar increases (T2).

- When water level in the distillation jar increases (L2).

- When water level in the cooling fluid reservoir decreases (L3).

- When temperature in the cooling fluid reservoir increases (T3).

- When water level in the product jar increases (L5).

All active safety control loops are implemented directly in the PLC. If any of mentioned breakdowns occur, the PLC deactivates specific actuators and disables user control of the model.

\section{COMMUNICATION}

Sensors and actuators described in the Chapter 3 are connected to PLC through AS-Interface bus (AS-Interface, 2011). This bus was designed to connect simple devices (distributed inputs and outputs) to PLC through special 2conductor yellow cable. All connected devices (except of several exceptions) are supplied by this cable as well. However, some modules need external power supply for its correct function (most often digital output modules). The external power is distributed by the second cable (often black). Nevertheless, this cable does not carry any data signal.

One function of the PLC was described in the Chapter 3. PLC also serves as a gateway between actuators and sensors and the supervisor controller. To connect to the supervisor system, the ModbusTCP protocol (The Modbus organization, 2005), based on the Modbus protocol has been chosen. While traditional Modbus protocol operates through serial line, the ModbusTCP can be run through TCP/IP networks. ModbusTCP belongs to the group of protocols implemented on the application layer of the OSI model. ModbusTCP does not need the checksum (unlike traditional Modbus), because the data protection is realized by lower OSI layers (Cisco Systems Inc., 2003). Figure 3 shows the communication scheme of the model.

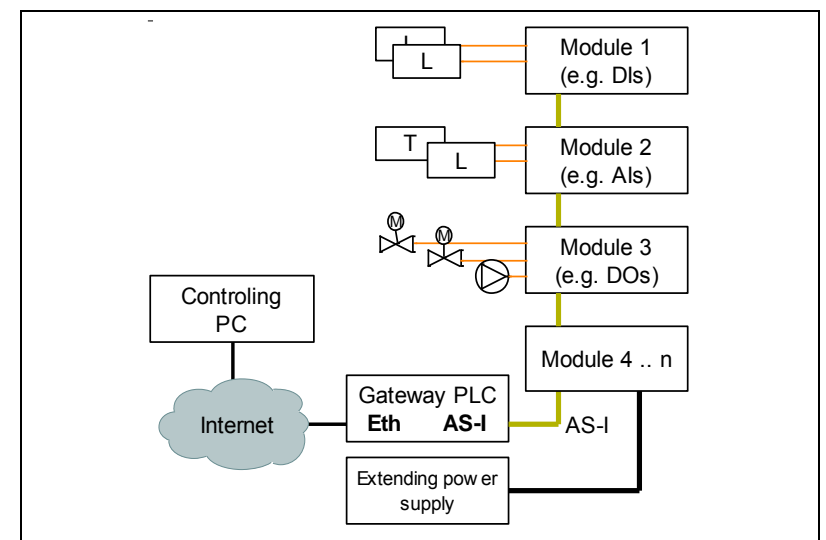

Fig. 3. Communication scheme of the model

\subsection{Mapping}

As mentioned above, PLC serves as a gateway between both networks. Two sets of ModbusTCP registers should be defined - one for reading and another for writing technological values. Each of them consists of 12 registers and every register is 16 bits long. Every analog value is transformed to 16 bits representation and thus occupies one register.

When the request for reading values is received, PLC creates the output frame by mapping all values to defined places in the output register and sends this frame to the supervisor system. If the writing request is received, each value of the input register is processed by defined algorithms - the value is compared with the current model state and if the action bound with value is considered as safe, it is performed. The model safety can not thus be influenced by the supervisor system designed by students.

\section{RESULTS AND FUTURE WORK}

The paper shows fundamental problems appearing during the project development and their solutions. Chapter 3 describes the chemical part and its safety problems. Chapter 4 shows the basic principle of two-steps communication. The model, when finished, should help students to acquire practice in the field of industrial automation. In the future, the automatic control algorithm for the PLC should be developed. Moreover, creating of the visualization ASP.NET server could be another possible project assignment.

\section{ACKNOWLEDGEMENTS}

This work was partially supported in by grant „Modern Methods and Approaches in Automation" from the Internal Grant Agency of Brno University of Technology (grant No. FEKT-S-11-6), Grant Agency of the Czech Republic (102/09/H081 SYNERGY - Mobile Sensoric Systems and Network) and Ministry of Education, Youth and Sports of the Czech Republic (Research Intent MSM0021630529 Intelligent systems in automation (VZ UAMT)

\section{REFERENCES}

Allbright, L. F. (2009). Albright's chemical engineering handbook, CRC Press, 978-0-8247-5362-7, Boca Raton

Cisco Systems Inc. (2003). Internetworking Technologies Handbook (4 $4^{\text {th }}$ Edition), Cisco press, 978-1587051197, San Jose

Churáček, J. (2007). Analytická separace látek, SNTL, 80-03-00569-8, Praha

*** (2011) http://as-interface.net - AS-Interface, Accessed on: 2011-08-15

*** (2005) http:/www.modbus.org - The Modbus organization, Accessed on: 2011-08-20 